Conclusion The patient was treat with Imiquimod 5\% cr. for 8 weeks with non significant results an partial vulvectomy (willingness of the patient) was performed.

\section{P2.56 A REMINDER FROM THE GREAT IMITATOR - GUMMATOUS SYPHILIS OF THE NASAL CAVITY WITH SEPTAL PERFORATION}

${ }^{1}$ IA George, ${ }^{1,2} \mathrm{HEL}$ Reno, 1,2 BP Stoner. 'Division of Infectious Diseases, Washington University, St Louis, MO, USA; ${ }^{2}$ Department of Anthropology, Washington University, St Louis, MO, USA

10.1136/sextrans-2017-053264.232

Introduction Gummatous syphilis presenting as nasal septal perforation is well described in the classic literature, but rarely encountered in the current antibiotic era. We present a man with a destructive nasal process with a delayed diagnosis of tertiary (late benign) syphilis.

Case Description A 45 year old Eritrean gentleman presented with an ulcero-nodular lesion of the left nares, progressive over the previous six months. He denied trauma or illicit drug inhalation. Exam was remarkable for left nasal cavity with an eroding destructive lesion perforating through the nasal septum and left nasal ala. He had no clinical signs or symptoms of neurosyphilis. Multiple biopsies revealed acuteon-chronic inflammation with focal necrosis and no evidence of malignancy. Fungal, treponemal and routine bacterial stains were negative, and tissue cultures were negative. Imaging indicated no bony destruction. The patient was treated for presumed cellulitis with multiple courses of oral antibiotics (cephalexin, amoxicillin) with no improvement in symptoms. At follow up, the patient tested negative for human immunodeficiency virus (HIV) infection and negative for anti-neutrophil cytoplasmic antibodies (ANCA). Serologic tests for syphilis were ultimately performed, revealing a rapid plasma reagin (RPR) titer of 1:512 with a reactive florescent treponemal antibody absorption test (FTA-ABS). A CSF evaluation was normal, with no pleocytosis and normal protein and glucose. Treatment was initiated with benzathine penicillin G, three doses of 2.4 million units each at one-week intervals. Clinical response to treatment is pending at the time of this report.

Discussion Gummatous syphilis is of clinical importance because of its potential for local destruction and disfigurement of the nasal structures. Early recognition and management has important individual and public health implications and this case would remind contemporary physicians that "the great imitator" could lurk behind unusual presentations.

\section{P2.57 A CASE OF DIFFICULT DIAGNOSIS: NEUROSYPHILIS IN HIV INFECTED PATIENT}

${ }^{1}$ Helena Biasizzo, ${ }^{2}$ Saša Simčiče; ${ }^{3} J a n e z$ Tomažič. ${ }^{1}$ General Hospital Novo Mesto, Novo Mesto - Slovenia; ${ }^{2}$ Institute if Microbiology and Immunology, Ljubljana - Slovenia; ${ }^{3}$ Clinic for Infectious Diseases and Febrille Illness, Ljubljana - Slovenia

\subsection{6/sextrans-2017-053264.233}

Introduction It is known that HIV infected patients with syphilis are more prone to invasion of $T$. pallidum into central nervous system. Moreover, the diagnosis of neurosyphilis in HIV infected patients can be particularly difficult and challenging since serological tests in the cerebrospinal fluid (CSF) can often be false negative.

Case report A previously healthy 40- year- old male was admitted to neurology department due to acute left side paresthesia, ataxia and diplopia. Since his head CT and CT-angiography scans were without pathological findings and his symptoms started recovering, he was treated conservatively. His symptoms worsened the next day and MR scan revealed right posterior pontine infarction. As part of routine screening for stroke in young patients, he was tested for HIV and syphilis. Serologic tests for syphilis were positive in serum as were screening and confirmatory tests for HIV infection. CD4+ cell count was $282 / \mathrm{mm}^{3}$ and HIV RNA was 9480 copies/ml. CSF analysis showed elevated protein level $(0,70 \mathrm{~g} / \mathrm{L})$ and lymphocytic pleocytosis (lymphocytes $30 / \mathrm{mm}^{3}$ ). CSF- RPR and CSFTPHA were not reactive. However, because of strong clinical suspicion of meningovascular syphilis, additional serological tests for syphilis in CSF, i.e. CSF-IgG-FTA-ABS and CSF-LIA (Line Immuno Assay), were performed: both were positive. After the confirmation of suspected meningovascular syphilis, treatment with intravenous benzylpenicillin was given for 21 days. Neurological symptoms subsided and patient was discharged with minimal neurological sequelae.

Conclusion The correct diagnosis of neurosyphilis in HIV infected patients presents a challenge since serologic tests can be false negative. Therefore, different serologic tests with high specifity and sensitivity should be used, newer tests such as LIA and CIA being particularly helpful. Clinicians should be aware of the characteristics of syphilis and HIV coinfection to establish the correct diagnosis and provide adequate treatment, which will minimise neurological impairments among these patients.

\section{LB 2.58 DRUG RESISTANCE MUTATIONS IN HUMAN IMMUNODEFICIENCY VIRUS TYPE 2 (HIV-2) STRAINS FROM PATIENTS IN GHANA}

${ }^{1}$ Christopher Zaab-Yen Abana, ${ }^{2}$ Sagoe Kwc, ${ }^{1}$ Bonney Ey ${ }^{3}$ Maina Ek, ${ }^{1}$ Aziati Id, ${ }^{1} E$ Agbosu, ${ }^{1} \mathrm{G}$ Mawuli, ${ }^{4}$ Styer Lm, 'Brandful Jam, 'Ampofo Wk. 'Virology Department, Noguchi Memorial Institute for Medical Research, College of Health Sciences, UG, Accra, Ghana; ${ }^{2}$ Department of Medical Microbiology, School of Biomedical And Allied Health Sciences, UG, Accra, Ghana; ${ }^{3}$ Centrefor Microbiology Research, Kenya Medical Research Institute, Kenya, Nairobi, Kenya; ${ }^{4}$ Bloodborne Viruses Laboratory, Wadsworth Centre, New York State Department of Health, Albany, New York, USA

\subsection{6/sextrans-2017-053264.234}

Introduction The Human Immunodeficiency virus (HIV) epidemic is characterised by the dominance of HIV type 1 (HIV1) worldwide. Consequently, antiretroviral therapy (ART) and drug resistance studies have focused almost exclusively on HIV-1. In Ghana both HIV-1 and HIV-2 co-circulate with lack of data on HIV-2 drug resistance mutations. We sought to determine drug resistance mutations in HIV-2 patients in Ghana.

Method We used purposive sampling to collect blood from 16 consented patients confirmed as HIV-2 and dual HIV-1/2 by serology and molecular assays. Real-time RT-PCR assay was used to determine the viral load of patients by using an HIV2 RNA International Standard from the National Institute for Biological Standards and Control (NIBSC). Nucleic acid (RNA and DNA) were extracted from plasma and peripheral blood mononuclear cells (PBMC) respectively. The reverse transcriptase (RT) and protease (PR) genes of HIV-2 were amplified 